\title{
EL VIDRIO ROMANO DE VENTANA EN LA BÉTICA. UNA APROXIMACIÓN A SU ESTUDIO A TRAVÉS DE LA ARQUEOMETRÍA
}

\author{
Almudena Velo Gala ${ }^{1}$
}

\begin{abstract}
Resumen:
La línea de investigación que presentamos en esta publicación surge de la ausencia de estudios sobre el vidrio y su aplicación como cierre de vanos en la arquitectura romana de la Bética. Siguiendo las últimas investigaciones realizadas sobre vidrio en la Antigüedad, nuestro trabajo contempla la importancia de aunar los datos arqueológicos con los aportados por el estudio arqueométrico de los materiales. El estudio de un relevante conjunto de fragmentos de vidrio plano documentados en la villa de Ronda del Marrubial (Córdoba, España) ha permitido la elaboración de una metodología de trabajo aplicable a otros ejemplos que hemos identificado en diversos yacimientos de la Bética. Los datos obtenidos permitirán elaborar conclusiones sobre los contextos a los que se asocian, su producción y comercio en el periodo romano en esta área geográfica.
\end{abstract}

\section{Palabras clave:}

Vidrio romano; vidrio de ventana; Bética; Córdoba; Ronda del Marrubial.

\begin{abstract}
:
The line of research that we present in this paper arises due to the lack of studies concerning the glass and its application as closing openings in Roman architecture of Hispania Baetica. Following latest researches about ancient glass, our study highlights the importance between archaeological and archaeometric studies. Our work commences with a relevant group of flat glass fragments documented in the villa of Ronda del Marrubial (Cordoba, Spain). This example will be the model study applied to other examples we have identified in several archaeological sites in Hispania Baetica. The answers will allow to draw conclusions about the contexts to which they are associated, their production and trade in this province in Roman period.
\end{abstract}

\section{Key words:}

Roman glass; window glass; Hispania Baetica; Cordoba; Ronda del Marrubial.

1 Departamento de Prehistoria y Arqueología, Universidad de Granada. 


\section{Introducción}

El vidrio romano de ventana es un material que aparece en numerosos yacimientos, bien en contextos de abandono o basureros, de forma muy fragmentada y en número reducido. Esto es debido a que este material, al igual que sucediera con otros de distinta naturaleza, era reaprovechado para su posterior reciclaje. El vidrio de ventana, dentro de las tipologías conocidas de vidrio, era muy apreciado para este fin, puesto que el grosor de estos primeros paneles aportaba una gran cantidad de vidrio para su posterior refundición. Este fenómeno explica, en gran parte, que el registro arqueológico de vidrio romano de ventana sea escaso, sobre todo en las provincias occidentales del Imperio, donde uno de los modelos de producción apunta a la instalación de industrias vidrieras dispersas y de pequeñas dimensiones donde primaría una producción de vidrio secundaria (Foster, Jackson, 2010: 3069), es decir, a partir de la refundición de bloques de vidrio en bruto y en la que el reciclaje tuvo un destacado papel.

Por otro lado, el desconocimiento generalizado de esta tipología de materiales entre investigadores y arqueólogos, ha dificultado el estudio de los mismos. Los fragmentos de vidrio plano no suelen ser identificados como tales en los listados de materiales y, en caso de serlo, son interpretados como restos de otras tipologías, concretamente recipientes (Ortiz Palomar, 2001a: 40). El estudio del vidrio romano de ventana, obliga a la revisión de todos aquellos fragmentos de vidrio depositados en almacenes y museos, lo cual, en ocasiones, es un tarea difícil de acometer. Además, consideramos que este material no ha contado con el interés que gozan otros, tales como la cerámica, a pesar de que la aplicación del vidrio para el cierre de vanos de ventana ha sido considerada como uno de los grandes avances en el empleo de este material. El menosprecio por parte de los investigadores se debe, principalmente, a que no proporcionan cronologías precisas, puesto que las técnicas de elaboración de los vidrios de ventana se mantuvieron invariables durante varios siglos. Debemos añadir que, aunque la mayoría de investigadores coinciden en el empleo de las distintas técnicas de fabricación en determinados periodos cronológicos, es posible que algunas de ellas no fuesen sustituidas del todo, por lo que pueden documentarse vidrios de ventana fabricados de diversa forma asociados a un mismo edificio y periodo (Foy, Fontaine, 2008: 430). Sin embargo, la importancia de su estudio radica en la información que nos proporcionan sobre las construcciones a las que se encuentran asociados, la funcionalidad de algunos espacios o sobre la producción y comercio de este tipo de materiales.

El empleo de técnicas arqueométricas está proporcionando en estos últimos años una información de gran interés para el conocimiento de la producción del vidrio en la Antigüedad. La ejecución de este tipo de análisis, como complemento a los estudios más tradicionales, constituye un factor de gran relevancia. Los datos 
proporcionados mediante dichas técnicas, tanto cualitativos como cuantitativos, de los componentes del vidrio, no sólo permiten conocer cuáles eran los materiales empleados y qué cantidad se utilizaba en su manufactura, sino que, además, aportan datos de interés sobre las fuentes de las que se extrajeron. La comparativa con otros materiales vítreos analizados contribuye a la identificación de los principales centros productores de vidrio en la Antigüedad (Freestone, 2005, 2006, cit. en Schiavon et al., 2012: 975). Por otro lado, estas técnicas también facilitan un conocimiento exhaustivo de los procesos de alteración del material de cara a afrontar nuevas técnicas de conservación.

\section{El vidrio romano de ventana}

Los primeros testimonios de esta tipología de vidrios se remontan a inicios del siglo I d.C., siendo muy probable que su descubrimiento tuviese lugar en torno al cambio de era y, concretamente, en la península itálica, puesto que los testimonios de vidrio de ventana en el área oriental son muy escasos y no se muestran en grandes cantidades hasta el siglo VI d.C. (Forbes, 1966: 187; Isings, 1971: 44; Foy, Fontaine, 2008: 409). En el caso de la península ibérica, los vidrios de ventana documentados han permitido fechar su utilización en Hispania desde la época de Tiberio (Ortiz Palomar, 2055: 44-45) hasta el siglo IV d.C., con una ausencia destacada a partir de esa centuria (Sánchez de Prado, 2006: 92). Su uso como cierre de vanos en este determinado momento pudo deberse a causa de una conjunción de varios fenómenos. Por un lado, el amplio desarrollo urbanístico que se inició a comienzos del Imperio, lo que propició el uso de técnicas constructivas novedosas y la creación de grandes estructuras de carácter público; dichas arquitecturas precisaban de la apertura de amplios orificios que favoreciesen una correcta iluminación (Fuentes Domínguez, 2001: 137). El vidrio se ofrecía como un material óptimo para satisfacer la mayor parte de las necesidades de este tipo de espacios, ya que, por sus cualidades, permitía la entrada de luz, preservaba de las inclemencias climáticas y, en cierta medida, posibilitaba la contemplación del paisaje. Por otro lado, el empleo de este material se encontraba en expansión debido al descubrimiento y la aplicación de la técnica del vidrio soplado, lo que provocó un descenso en sus costes de fabricación y un aumento en su producción. El vidrio, que hasta entonces había sido un material de lujo reservado a unos pocos por su elevado coste, es a partir de ese momento adquirido por todos los estratos sociales (Vigil, 1967: 85; Fuentes Domínguez, 2001: 157).

Aunque su presencia está sobre todo asociada a edificios de carácter público $y$, en especial a las salas calientes de conjuntos termales, es frecuente documentar estos materiales en ambientes de carácter privado, tales como villae o domus. La funcionalidad de los paneles de vidrio en los baños públicos y privados era facilitar 
la iluminación de los espacios, contribuir al mantenimiento de la temperatura en las salas calientes, tepidarium y caldarium y, de este modo, favorecer el ahorro de combustible en los hornos. En cambio, en ambientes privados, puede observarse una selección de los vanos cubiertos con este material, los cuales se corresponden con las estancias de mayor prestigio del conjunto, sobre todo en los triclinia. Es por ello que algunos investigadores plantean que se uso, además de cómo un elemento de confort, está considerado como un elemento de distinción social (Vipard, 2009: 9). Sin embargo, no podemos olvidar que el vidrio no fue el único elemento empleado para este fin, puesto que con anterioridad otros materiales cumplieron tal función, en especial el yeso especular o lapis specularis. En Hispania el vidrio de ventana debió tener en el lapis specularis un fuerte competidor, puesto que las minas de yeso especular más importantes del Imperio se hallaban en la Hispania Citerior. Esto pudo condicionar en buena medida el uso del vidrio para la cubrición de vanos, lo que habría llevado a un predominio de la aplicación de láminas de yeso durante el periodo en el que la explotación del mismo se mantuvo en auge (Fuentes Domínguez, 2001: 138-139).

\section{Antecedentes sobre el estudio de vidrio de ventana en la península ibérica}

A nivel nacional, los estudios referentes al vidrio de ventana en periodo romano más concluyentes han sido los realizados en el marco de la Universidad de Zaragoza, concretamente los ejecutados por los investigadores E. Ortiz Palomar y J.Á. Paz Peralta. Hay que destacar que estos trabajos deben considerarse pioneros en la identificación de una de las tipologías de vidrio de ventana: el vidrio hemisférico circular (Ortiz Palomar, Paz Peralta, 1997), del que actualmente se conocen numerosos ejemplos en diversas zonas del Imperio. Al margen de los hallazgos recopilados por estos autores, para el resto de la península ibérica disponemos, en la actualidad, de información escasa y dispersa. En general, el vidrio de ventana suele aparecer mencionado, entre otros objetos, en estudios referentes a colecciones y de manera individual en muy pocas publicaciones. Algunas de estas excepciones, al margen de las investigaciones señaladas anteriormente, son el estudio de los vidrios de ventana documentados en el campamento romano de Cidadela, (La Coruña) publicado por J.M. Caamaño y $M^{a}$ A. Vázquez en el año 2001, en Guadix, publicado por I. Cambil Campaña en el año 2012, o el estudio de los vidrios hallados en el yacimiento de Cortalago (Minas de Riotinto, Huelva,) publicado el mismo año por M. C. Regalado Ortega et al. (2012).

Llama la atención que la mayoría de los testimonios que se conocen de esta tipología de material procedan de la franja septentrional de la península ibérica, lo cual nos lleva a pensar que en otras áreas peninsulares esta ausencia sea debida a la escasez de estudios específicos sobre dicho material. Por otra parte, 
las condiciones climatológicas existentes en las regiones más norteñas podrían haber sido un factor condicionante que justificara por sí mismo un mayor uso del vidrio de ventana en las mismas, pero el progreso en la investigación arqueológica en zonas meridionales del Imperio ha conducido a refutar esta idea inicial (Foy, Fontaine, 2008: 408).

Finalmente, resaltar el hecho de que no existen estudios arqueométricos específicos sobre vidrio romano de ventana en la Península, aunque sí tenemos algunos datos interesantes en materiales analizados como parte del estudio arqueométrico global de alguna colección.

\section{El conjunto de vidrios planos de la villa de Ronda del Marrubial (Córdoba)}

Nuestro trabajo de investigación comienza con el estudio de un conjunto relevante de 219 fragmentos de vidrio plano de ventana, asociados a una villa de época romana, que se documentaron en el transcurso de las excavaciones realizadas en el año 2008 en un solar ubicado en Ronda del Marrubial esquina Avd. Agrupación de Córdoba (Córdoba). Actualmente se conoce esta edificación bajo la denominación de "villa de Ronda del Marrubial".
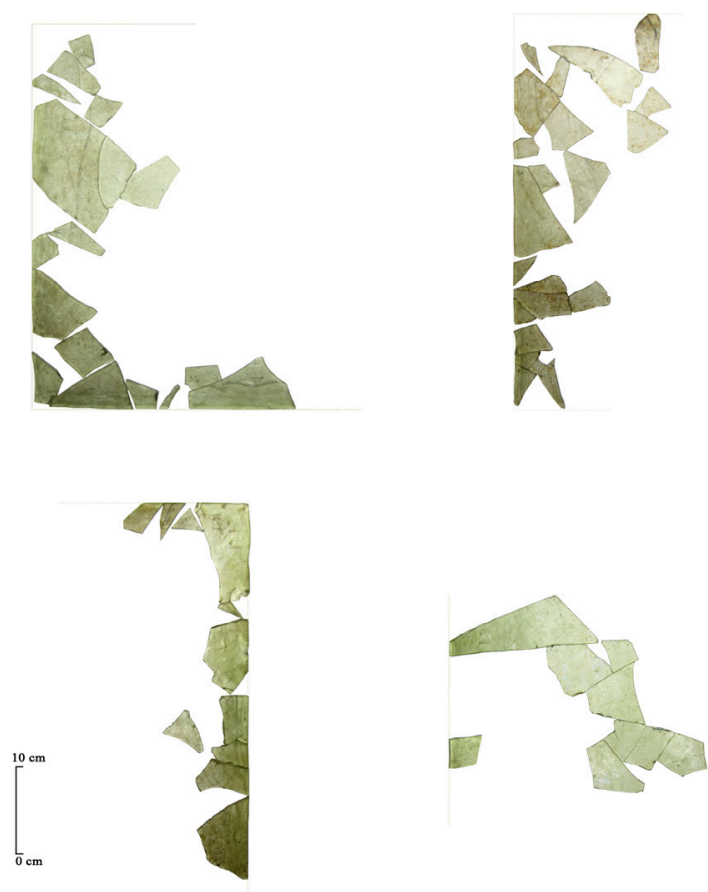

Fig. 1 Reconstrucción de los paneles (A. Velo Gala). 
Según los datos del informe elaborado por la directora de la excavación, podemos fechar los paneles de la villa de Ronda del Marrubial entre los siglos I-III d.C., periodo al que pertenecen la mayor parte de las estructuras (Ortiz Ramírez, 2011: 273-274). Las restituciones que hemos efectuado han permitido distinguir un mínimo de cuatro unidades, que alcanzarían unas medidas aproximadas de 44 y $46 \mathrm{~cm}$, no siendo posible determinar si se trata de paneles cuadrados o de forma rectangular al no existir los dos laterales anexos restituidos (Fig. 1). Por su proximidad, podrían asociarse a los vanos de ventana de una estancia identificada como un posible triclinium. Sin embargo, tampoco se descarta su vinculación con otros espacios como un atrio, ubicado junto al triclinium, siguiendo un esquema similar al de pórticos con ventanas que se conocen en diversas domus del altoimperio (Vipard, 2003). Sí se confirma que su presencia está estrechamente ligada a las estancias o zonas de más prestigio de la vivienda, al igual que sucede con otros vidrios romanos de ventana pertenecientes a ambientes de carácter privado.

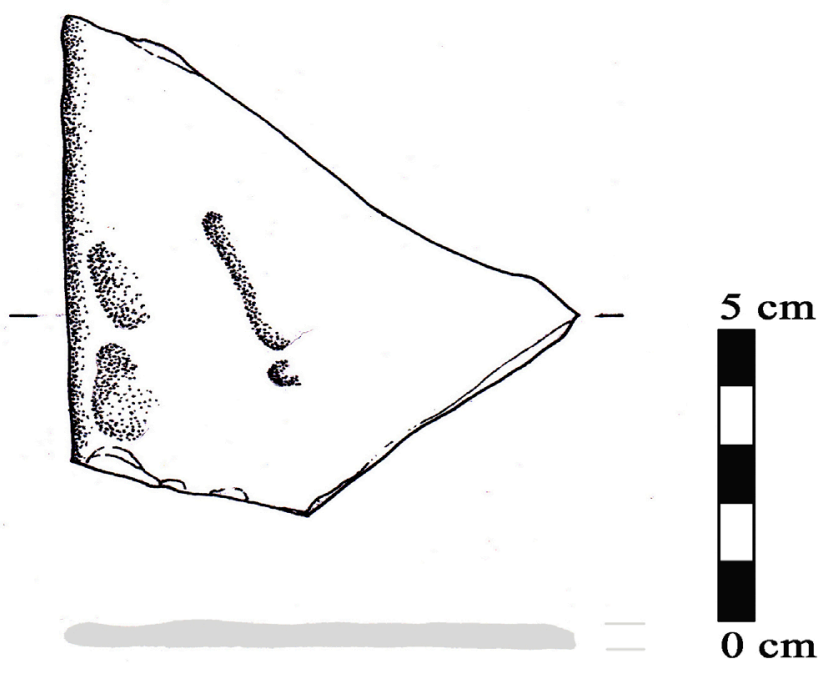

Fig. 2 Impronta de las herramientas empleadas para el estirado de la masa vítrea (A. Velo Gala).

El examen visual de cada uno de los fragmentos permitió constatar una serie de características comunes en todos ellos. Se tratan de vidrios de gran grosor, entre 1-7 mm, siendo las zonas más próximas a los bordes las de mayores dimensiones. Es en esta franja donde puede observarse la impronta producida por el uso de herramientas para el estirado de la masa vítrea (Fig. 2). Son vidrios translúcidos 
de colores "sucios" que oscilan entre los tonos ámbar-verde-azul, a causa de una escasa depuración de impurezas en la masa vítrea. Muestran dos superficies diferenciadas, una mate lisa y otra brillante e irregular. Esta distinción es una característica esencial para la identificación de la técnica de elaboración de esta tipología de vidrio: el vidrio colado y estirado sobre una superficie o molde. La superficie mate y lisa es la que se corresponde con el lado que estuvo en contacto con otra superficie, mostrando la impronta que le transfirió durante este proceso; en este caso, es posible que el vidrio fuese vertido sobre una placa o molde de piedra, arcilla u otro material cubierto con partículas de arena que evitasen su adhesión, tal y como denotan las marcas visibles en forma de picadura. El lado superior, irregular y brillante, es el resultado del vertido del vidrio y de la propia naturaleza de este material (Ortiz Palomar, Paz Peralta, 1997: 438; Dunn, 1986: 6, cit. en Ortiz Palomar, 2001a: 38; Ortiz Palomar, 2001b: 350; Foy, Fontaine, 2008: 409). Esta técnica de fabricación, aunque no existe ninguna referencia escrita de época clásica, era el sistema inicial para la obtención de láminas vítreas en el periodo romano (Vigil, 1967: 107; Ortiz Palomar, 2001a: 36), predominando desde el siglo I d.C. hasta el III d.C. (Foy, Fontaine, 2008: 409).

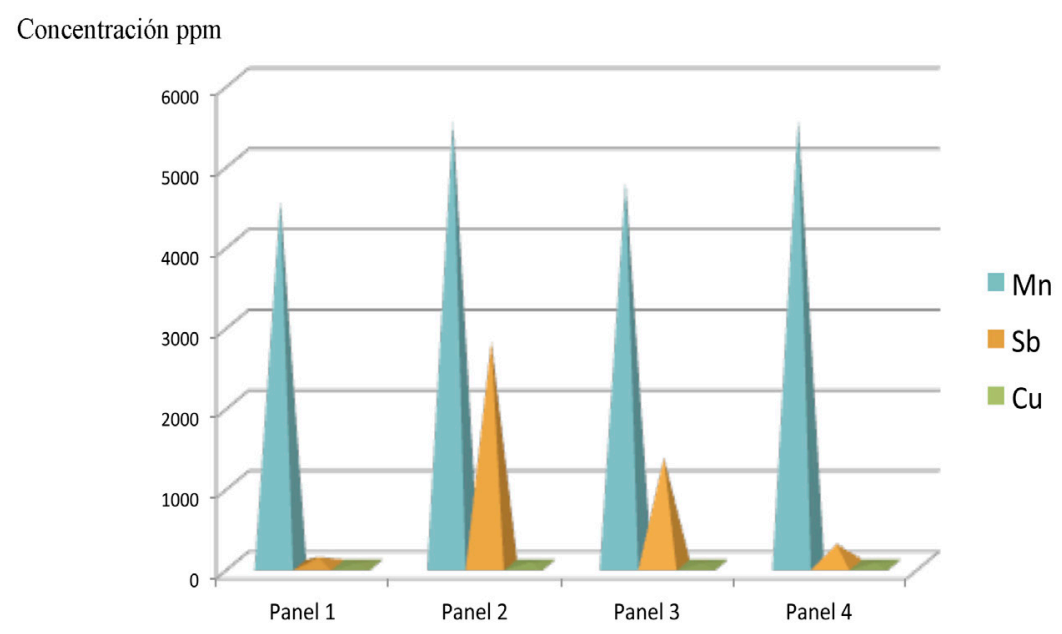

Fig. 3 Concentración en ppm de los elementos traza que determinan la práctica del reciclaje de los paneles (análisis ICP-MS).

Como complemento a los datos recopilados en esta primera fase de estudio, se realizó una toma de muestras representativas de cada uno de los paneles identificados para la ejecución de un análisis físico-químico. 
Para la ejecución de los análisis físico-químicos se han seleccionado las siguientes técnicas: microscopia electrónica de barrido (SEM) y microanálisis por espectroscopía de energía dispersiva de rayos $\mathrm{X}$ (EDS), fluorescencia de rayos $\mathrm{X}$ (XRF) y espectrometría de masas con fuente de plasma de acoplamiento inductivo (ICP-MS).

Los primeros resultados obtenidos para las concentraciones de los elementos primarios del vidrio, permitieron confirmar que los porcentajes se encuentran dentro de los estipulados para los vidrios de época romana, vidrios silicato sódico cálcicos. En cambio, las medidas de los elementos traza en los fragmentos analizados han revelado que la mayoría de ellos están presentes en las muestras de forma no intencionada; tan sólo el manganeso y el antimonio presentan valores indicativos que confirmarían su adición. Las altas concentraciones de estos elementos muestran que los paneles de vidrio de la villa de Ronda de Marrubial fueron elaborados siguiendo una práctica muy común de este periodo: el reciclaje (Fig. 3). Estos elementos forman parte del óxido de manganeso y del óxido de antimonio, agentes que se emplearon para la decoloración del vidrio durante el periodo romano, predominando el empleo de este último a partir del siglo III d.C. (Ortiz Palomar, 2001: 20).

Respecto al estado de conservación de los vidrios tenemos que destacar que el conjunto se halló en un excelente estado de conservación, en comparación con el estado en el que suelen aparecer estos materiales en las excavaciones. Las imágenes obtenidas mediante SEM permitieron apreciar con mayor precisión la delaminación y microfisuración de las primeras capas del vidrio, degradaciones que se producen en las fases iniciales del deterioro del vidrio (Fig. 4).

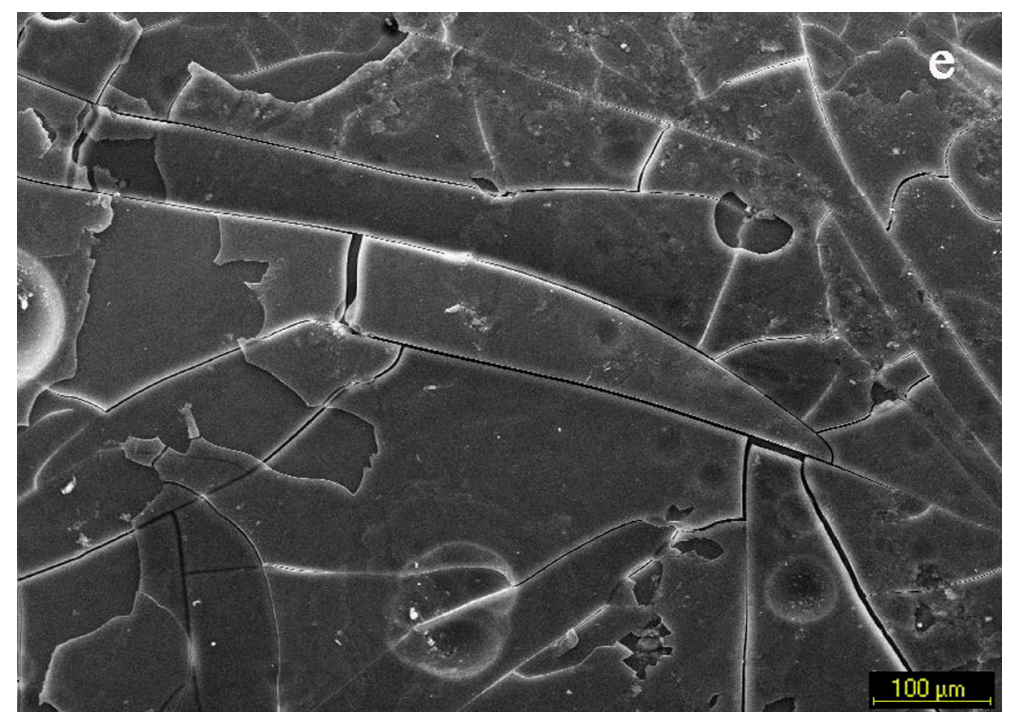

Fig. 4 Deterioro en la superficie del vidrio (microfisuración y delaminación) (SE). 


\section{Conclusiones}

Los resultados obtenidos hasta ahora sobre el vidrio de ventana en la arquitectura romana de la Bética, nos permiten postular que su presencia en yacimientos de esta cronología es más abundante de lo estipulado hasta el momento.

Puesto que los vidrios de ventana presentan una serie de características comunes a todos ellos derivadas de su proceso de elaboración (vidrio colado o vertido y estirado sobre una superficie o molde), queremos incidir en la relevante información que se deriva de los estudios arqueométricos. La composición química del vidrio proporciona datos de un gran interés acerca de la procedencia de los paneles y sobre las posibles variaciones en su manufactura a lo largo de las centurias.

El excepcional conjunto de fragmentos que se documentaron en las excavaciones de la villa de Ronda del Marrubial (Córdoba) denota la importancia del uso de estos materiales en las estancias más destacadas de las arquitecturas privadas. Aunque los primeros datos arrojados por el estudio arqueométrico ofrecen una información de gran relevancia no podemos determinar con exactitud cuál es su procedencia. El estudio comparativo de los análisis físico-químicos con otros materiales de la capital y de otros yacimientos de la Bética permitirá establecer hipótesis sobre la manufactura y comercio de esta tipología de vidrio.

Finalizamos este trabajo incidiendo en la importancia del valor que tiene esta tipología de materiales, los cuales no son tenidos en cuenta a la hora de realizar el estudio de los contextos donde se han documentado. Aunque, como ya mencionamos, no permiten fechar un estrato con la precisión que permiten otros materiales, cuando se documentan asociados a arquitecturas aportan una información detallada sobre la morfología de las mismas, puesto que en contadas ocasiones contamos con la conservación del alzado de las estructuras hasta los niveles de ventana. Además, no debemos olvidar que se trata de un material que debe ser considerado el predecesor de una de las manifestaciones artísticas del Medievo: la vidriera.

\section{Bibliografía}

Caamaño, J. M.; Vázquez Ma A.: "El vidrio de ventana de época romana hallado en el campamento de Cidadela (Sobrado dos Monxes, A Coruña). Catálogo de piezas", Gallaecia, no 20, 2001, 205-216.

Cambil Campaña, I.: "Vidrio romano en Guadix: el vidrio de ventana", Boletín del Centro de Estudios Pedro Suárez: Estudios sobre las comarcas de Guadix, Baza y Huéscar, nº 25, 2012, 47-62. 
Forbes, R. J.: "Glasses". Studies in ancient technology vol. V, (1966), 110-231.

Foy, D.; Fontaine, S. D.: "Diversité et evolution du vitrage de l'Antiquité et du haut Moyen Âge", Gallia, 65, 2008, 405-459.

Fuentes Domínguez, A.: "El vidrio y su uso en la Arquitectura", Vidrio Romano en España. La revolución del vidrio soplado, Segovia, 2001, 136-139.

Isings, C.: Roman glass in Limburg, Gröningen, 1971.

Ortiz Palomar, E.: "Definición, tecnología y fabricación del vidrio antiguo", Vidrio romano en España. La revolución del vidrio soplado, Segovia, 2001a, 9-60.

Ortiz Palomar, E.: Vidrios procedentes de la provincia de Zaragoza: El Bajo Imperio Romano, Zaragoza, 2001b.

Ortiz Palomar, E.: "Vitrages d'époque romaine provenant d Espagne", en Vitres de I'Antiquité, Catalogue d'exposition, Bavay, 2005, 44-46.

Ortiz Palomar, E.; Paz Peralta, J. Á.: "“El vidrio en los baños romanos", Termalismo antiguo. Actas del I Congreso Peninsular (Arnedillo, La Rioja, 1996), Madrid, 1997, 437-452.

Ortiz Ramírez, L.: "Una villa romana en Ronda del Marrubial del s. I al IV d.C.", Romvla, 10, 2011, 253-276.

Regalado Ortega et al.: "Vidrios romanos de ventana del yacimiento de Cortalago (Minas de Riotinto, Huelva)", Acta do V encuentro de Arqueología peninsular, 2012, 765-772.

Sánchez de Prado, M D. (2006): "Los materiales de vidrio", La villa de Cornelivs, Valencia,

Schiavon et al:: "A combined multi-analytical approach for the study of Roman glass from south-west Iberia: Synchrotron $\mu$-XRF, external PIXE/PIGE and BSEM-EDS", Archaeometry, 54, 6, 2012, 974-996.

Vigil, M.: El vidrio en el mundo antiguo, 1967.

Vipard, P.: "Les portiques fenêtrés dans les domus du Haut-Empire romain", en Bulletin archéologique du CTH5: Antiquité, Archéologie classique, fasc. 30, 2003, Paris, 99-

Vipard, P:"L'usage du verre à vitre dans I'architecture romaine du Haut Empire", Verre et Fênetre de I'Antiquité au XVIIle siècle. Actes du premier colloque international de l'association VERRE \& HISTOIRE (Paris-La Défense / Versailles 13- 14-15 octobre 2005), 2009, Paris, 3-10. 